DOI:10.5216/cab.v15i1.13763

\title{
AVALIAÇÃO ULTRASSONOGRÁFICA DOS EFEITOS DA ACUPUNTURA NA INVOLUÇÃO UTERINA EM CADELAS
}

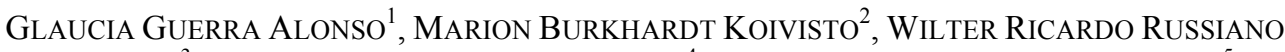 \\ VICENTE $^{3}$, Silvia Helena Venturoli PERRI ${ }^{4}$, MARCus ANTÔNIO Rossi FELICIANO ${ }^{5}$ \\ ${ }^{1}$ Pós-Graduanda da Universidade Estadual Paulista, Araçatuba, SP, Brasil. \\ ${ }^{2}$ Professora Doutora da Universidade Estadual Paulista, Araçatuba, SP, Brasil. \\ ${ }^{3}$ Professor Doutor Universidade Estadual Paulista, Jaboticabal, SP, Brasil \\ ${ }^{4}$ Professora Doutora da Universidade Estadual Paulista, Araçatuba, SP, Brasil. \\ ${ }^{5}$ Doutor Jovem Pesquisador da Universidade Estadual Paulista, Jaboticabal, SP, Brasil. \\ marcusfeliciano@yahoo.com.br
}

O objetivo deste estudo foi avaliar, por meio da ultrassonografia, os efeitos da acupuntura na involução uterina, durante período puerperal eutócico em cadelas, com estimulação elétrica (eletroacupuntura). Estudou-se o fenômeno da involução uterina eutócica, em 16 fêmeas divididas aleatoriamente em dois grupos de oito animais cada, em que o grupo 1 (G1) recebeu tratamento com estimulação elétrica (eletroacupuntura) da agulha nos pontos BP6, F3, Bai Hui, VC1, B23 e B26, e o grupo 2 (G2), grupo controle, recebeu tratamento em pontos Sham (pontos falsos). O acompanhamento do fenômeno da involução uterina foi realizado por ultrassonografia convencional, nos dias 0 (dia do parto), 1, 3, 7 e 14 pósparto. Utilizou-se a avaliação estatística pelo método SAS, com o propósito de comparar os grupos. Os resultados dos exames ultrassonográficos (média e desvios padrões em centímetros) para o período próprio de observação foram, respectivamente: $\mathrm{G} 1: 0,30 \pm 0,01(\mathrm{P}) ; 2,08 \pm 0,21(\mathrm{LU})$; $4,13 \pm 0,23(\mathrm{DU})$ e $\mathrm{G} 2: 0,31 \pm 0,01(\mathrm{P}) ; 1,82 \pm 0,09(\mathrm{LU})$; $3,92 \pm 0,11$ (DU). Pôde-se concluir que a eletroacupuntura apresentou efeitos sobre o tônus uterino (duradouros e uniformes ao final do tratamento). A eletroacupuntura infere positivamente no tônus uterino, corroborando no auxílio da prevenção de transtornos puerperais.

PALAVRAS-CHAVE: cadela; eletroacupuntura; involução uterina; pós-parto; ultrassonografia.

\section{ULTRASOUND EVALUATION OF ACUPUNCTURE EFFECTS ON UTERINE INVOLUTION IN BITCHES}

\section{ABSTRACT}

The aim of this study was to evaluate, by means of ultrasound exam, acupuncture effects on uterine involution during the postpartum period in bitches with electroacupuncture. The phenomenon of eutocic uterine involution was studied in 16 bitches, randomly divided into two groups of eight animals each. Group 1 (G1) was treated with electric stimulation (electroacupuncture) of the needle at the points SP6, L3, Bai Hui, TB1, B23, B26, and group $2(\mathrm{G} 2)$, the control group, received treatment in Sham points (placebo points), also with electrical stimuli.
The monitoring of the phenomenon of uterine involution was performed by ultrasonography on days 0 (partum), 1, 3, 7 and 14 postpartum. We used SAS statistical analysis method, in order to compare the groups. The results of ultrasound examinations (mean and standard deviations in $\mathrm{cm}$ ) for the period of observation were as follows: G1: $0.30 \pm 0.01(\mathrm{P}), 2.08 \pm 0.21(\mathrm{LU}), 4.13 \pm 0.23(\mathrm{DU})$ and G2: $0.31 \pm 0.01(\mathrm{P}) 1.82 \pm 0.09$ (LU), $3.92 \pm 0.11$ (DU). We concluded that electroacupuncture affected uterine tone (lasting and uniform at the end of treatment). 
Electroacupuncture produces a positive tone in the uterus, aiding at the prevention of puerperal diseases.

KEYWORDS: bitch; electroacupuncture; uterine involution; postpartum; ultrasound.

\section{INTRODUÇÃO}

A acupuntura tem vasto uso no tratamento de afecções, tanto em seres humanos como em animais. Como qualquer método terapêutico, possui limitações e sucessos, mas a quase inexistência de efeitos colaterais, o baixo custo e a fácil administração da terapêutica fazem com que a Medicina Tradicional Chinesa (MTC) seja uma opção para auxiliar na prevenção de transtornos reprodutivos (1).

Em ginecologia e obstetrícia humana, utiliza-se a acupuntura em mulheres devido à técnica apresentar efeito analgésico e atuar sobre a motilidade uterina, sendo útil no trabalho de parto e no tratamento de cólicas menstruais, além de normalizar as funções endócrinas. A acupuntura também é utilizada em mulheres lactantes, para diminuição da produção de secreção láctea (2). Em medicina veterinária, não há relato da utilização dessa técnica medicinal no tratamento ou prevenção de afecções reprodutivas.

As afecções reprodutivas observadas em medicina veterinária são a retenção de placenta, sub-involução uterina, atonia e endometrites, afecções que podem comprometer o estado reprodutivo dos animais (3). Para tanto, espera-se que, da mesma forma que na medicina, a utilização da acupuntura pode ser de grande valia no auxilio e controle dessas enfermidades.

Entre as mais importantes doenças, a piometra, caracterizada pela inflamação do útero com acúmulo de exsudato, é uma das enfermidades mais comuns do trato genital das fêmeas caninas $(4,5)$. Rotineiramente, o melhor método para se realizar o diagnóstico de piometra em caninos é por meio da ultrassonografia, na qual os achados de imagem típicos incluem aumento de volume uterino, evidenciado pela imagem do útero contorcido com seu interior repleto de líquido anecoico ou hipoecoico, geralmente homogêneo, porém, em alguns casos, é possível observar floculações e a parede endometrial pode estar espessada ou não. Ademais, a dilatação uterina causada pela piometra é vista durante o exame de ultrassom como múltiplas estruturas circulares (cortes transversais dos cornos uterinos justapostos) ou estruturas tubulares (corte sagital). A parede do órgão pode estar visivelmente espessada; entretanto, a cronicidade da afecção e o grau de distensão da parede uterina (presença de muito conteúdo) pode torná-la mais delgada (6-8).

$\mathrm{O}$ presente estudo tem como objetivo avaliar, por meio do exame ultrassonográfico, os efeitos da acupuntura na involução uterina, durante o período puerperal eutócico em cadelas, com a estimulação elétrica (eletroacupuntura).

\section{MATERIAL E MÉTODOS}

Foram utilizadas, neste experimento, 16 cadelas prulíparas, pós-parto eutócico, com idade entre 2 a 8 anos de vida e de raças variadas (sem raça definida, Poodles, Cocker Spaniels e outras), pacientes do Setor de Obstetrícia da UNESP/Jaboticabal. Os animais foram submetidos a exame físico, obstétrico e perfil laboratorial (hemograma, perfil bioquímico e urinálise) para avaliação do estado de saúde. Após avaliação clínica e seleção dos animais hígidos, as cadelas foram divididas aleatoriamente em dois grupos experimentais (Grupo 1-G1: 8 animais tratados com eletroacupuntura; e Grupo 2 - G2: controle, 8 animais submetidos à falsa acupuntura).

Os pontos de acupuntura avaliados foram o BP6, F3, B23, B26, Bai Hui e o VC1. O ponto BP6 (Baço/Pâncreas) localiza-se a três "tsun" (corresponde em cão, à distância interfalangeana do terceiro dedo em membro torácico) proximal à extremidade do maléolo medial, na margem medial distal da tíbia (Figuras 1A e 2). O F3 (Fígado) corresponde à extremidade distal do membro pélvico, em face craniomedial, em terço proximal do $2^{\circ}$ osso metatarsiano, entre o $1^{\circ}$ e $2^{\circ}$ ossos metatarsianos (Figuras 1B e 2). O ponto B23 (Bexiga) localiza-se um e meio "tsun" lateral à linha média, na horizontal traçada abaixo do processo espinhoso da $2^{\circ}$ vértebra lombar (Figuras 1C e 3). O B26 (Bexiga) está localizado um e meio "tsun" lateral à linha média, em linha horizontal traçada ventralmente ao processo espinhoso da $5^{\circ}$ vértebra lombar (Figuras $1 \mathrm{C}$ e 3 ). O ponto Bai Hui é lombossacro, sendo localizado em linha média dorsal dessa junção, entre L7 e S1 (Figuras 1C e 
3). $\mathrm{O} \mathrm{VC} 1$, relacionado à linha media do corpo, Os pontos falsos estão dispostos $1-2 \mathrm{~cm}$ encontra-seno centro do períneo, entre o ânus e a lateralmente aos pontos verdadeiros. comissura vulvar posterior (Figura 1C) $(9 ; 10 ; 11)$.

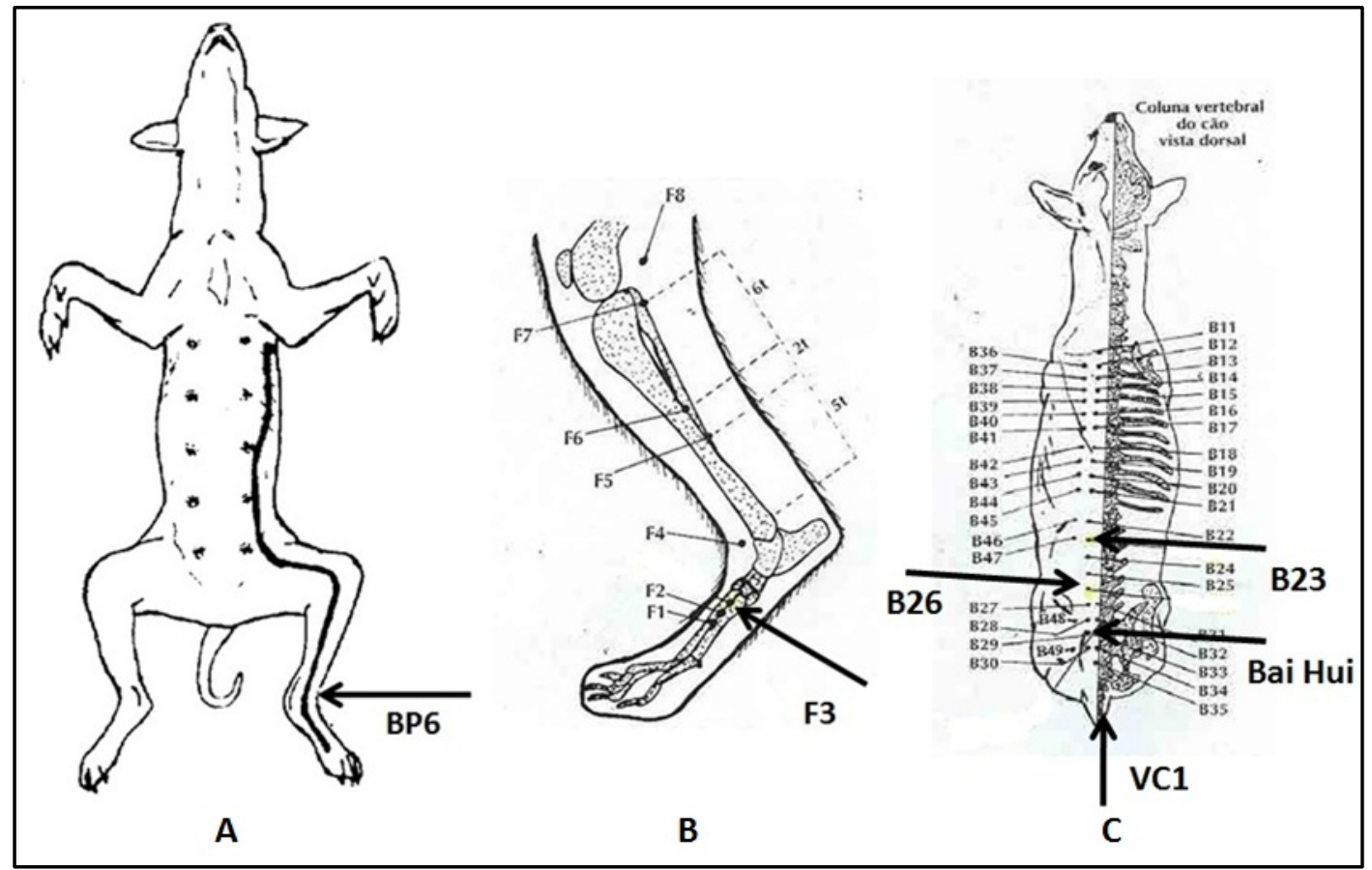

Figura 1: A) Esquema de um cão em posição ventro-dorsal, meridiano do Baço / Pâncreas. A seta indica o ponto de acupuntura BP6, três "tsun" proximal à extremidade do maléolo medial, face interna do segmento distal da tíbia; B) Esquema do segmento distal do membro pélvico de cão, face crânio-medial, meridiano do Fígado. Ponto de acupuntura F3, no terço proximal do $2^{\circ}$ metatarsiano, entre o $1^{\circ}$ e $2^{\circ}$; C) Esquema da região dorsal de um cão, meridiano da Bexiga. Pontos de acupuntura B23 (1 e 1/2 "tsun" lateral à linha média, na horizontal traçada abaixo do processo espinhoso da $2^{\circ}$ vértebra lombar), B26 (na horizontal traçada abaixo do processo espinhoso da $5^{\circ}$ vértebra lombar), Bai Hui (ponto lombo-sacro, localizado na linha média dorsal, entre L7 e S1) e VC1 (no centro do períneo, entre o ânus e a comissura vulvar posterior) (9).

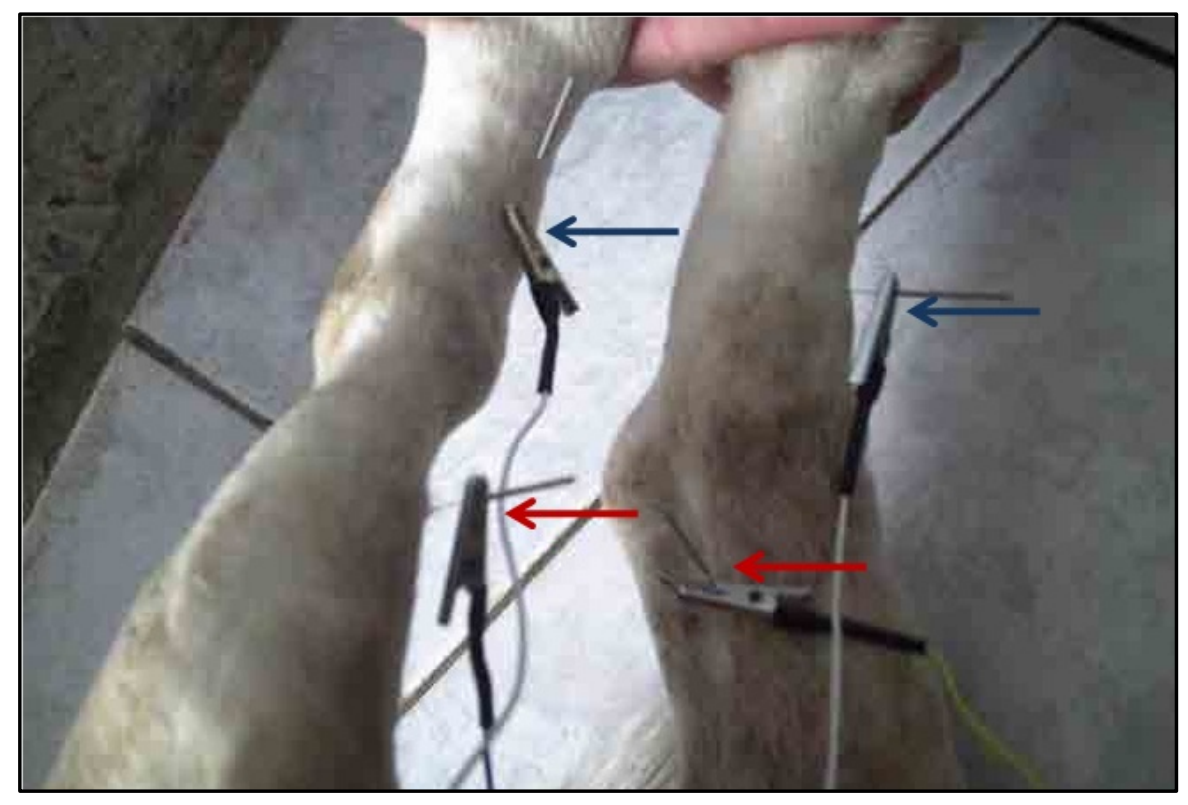

Figura 2: Vista medial dos membros pélvicos de cão, ilustrando os locais que receberam os estímulos 
elétricos em BP6 (seta vermelha) e F3 (seta azul), durante sessão de acupuntura.

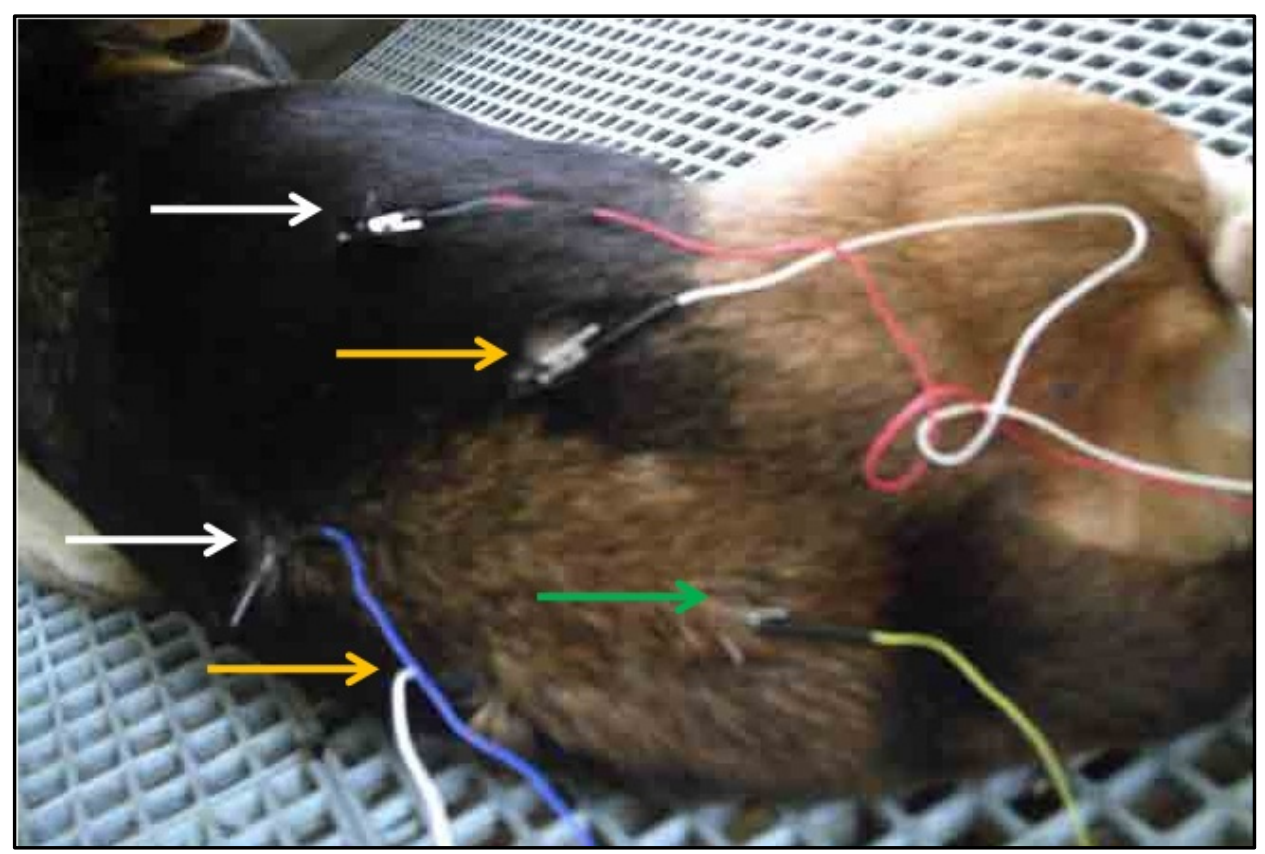

Figura 3: Vista dorsal de cão, ilustrando animal que recebeu estímulos elétricos em B23 (seta branca), B26 (seta amarela) e Bai Hui (seta verde), durante sessão de acupuntura.

A eletroacupuntura foi realizada por meio da conexão de eletrodos, do aparelho de eletroacupuntura, às agulhas de acupuntura previamente inseridas nos pontos e falsos pontos de acupuntura. Utilizaram-se aparelho de eletroacupuntura da marca SIKURO e agulhas chinesas da marca SUZHOU HUANQIU (Acupuncture and Medical Appliance), filiformes cilíndricas, compostas de aço inoxidável estéreis e descartáveis e com conteúdo reduzido de níquel para minimizar possíveis reações alérgicas. A espessura das agulhas variou entre 0,22 a $0,45 \mathrm{~mm}$.

A estimulação elétrica foi realizada bilateralmente, no Sinal 3 (modo denso disperso, trem de pulsos mistos), frequência de 5 e $15 \mathrm{~Hz}$, a cada 3 segundos, por 10 minutos. Conectaram-se os pontos de acupuntura BP6 ao B23, F3 ao B26 e Bay Hui ao VC1. Os animais eram contidos mecanicamente em decúbito lateral (independente de ser direito ou esquerdo), para a introdução das agulhas (bilateralmente) após prévia anti-sepsia da pele com álcool $70 \%$. Após conexão dos eletrodos, iniciou-se o estímulo por meio de corrente elétrica por 10 minutos (Figuras 2 e 3 ).

Realizou-se a eletroacupuntura e falsa eletroacupuntura nas cadelas por 10 minutos nos dias 1,3 e 7 pós-parto eutócico. Os exames ultrassonográficos foram realizados nas cadelas no dia do parto e uma hora após os animais serem submetidos à acupuntura, nos dias $1,3,7$ e 14 pósparto.

Para realização da ultrassonografia nas cadelas, utilizou-se aparelho marca PIE MEDICAL SCANNER 200, com transdutor convexo de 5,0 e $7,5 \mathrm{MHz}$. As imagens obtidas no experimento foram registradas em impressora específica acoplada ao aparelho. Foram avaliadas, por meio desse exame, a espessura da parede, lúmen e diâmetro, em porção caudal de ambos cornos uterinos. O corte ultrassonográfico realizado para a avaliação das características uterinas foi o transversal (Figura 4).

Antes dos exames ultrassonográficos (24 horas), administrou-se dimeticona, na dosagem de oito gotas por animal. Para realização do exame ultrassonográfico, realizou-se também a tricotomia ampla da região abdominal e utilizou-se uma espessa camada de gel condutor no local do exame (12).

$\mathrm{O}$ delineamento experimental foi inteiramente casualizado. Os dados foram previamente testados para normalidade dos resíduos e homogeneidade das variâncias (teste F). Foi utilizado o PROC MEANS-SAS®.

As médias reais ou transformadas foram avaliadas pela análise de variância e, em caso de significância, foi realizado o desdobramento do estudo estatístico de médias pelo teste pareado de médias (teste $\mathrm{t}$ ). As médias não homogêneas em teste de normalidade foram avaliadas por meio do teste 
Mann-Whitney. Foi utilizado nível de significância de 5\% para todos os testes realizados.

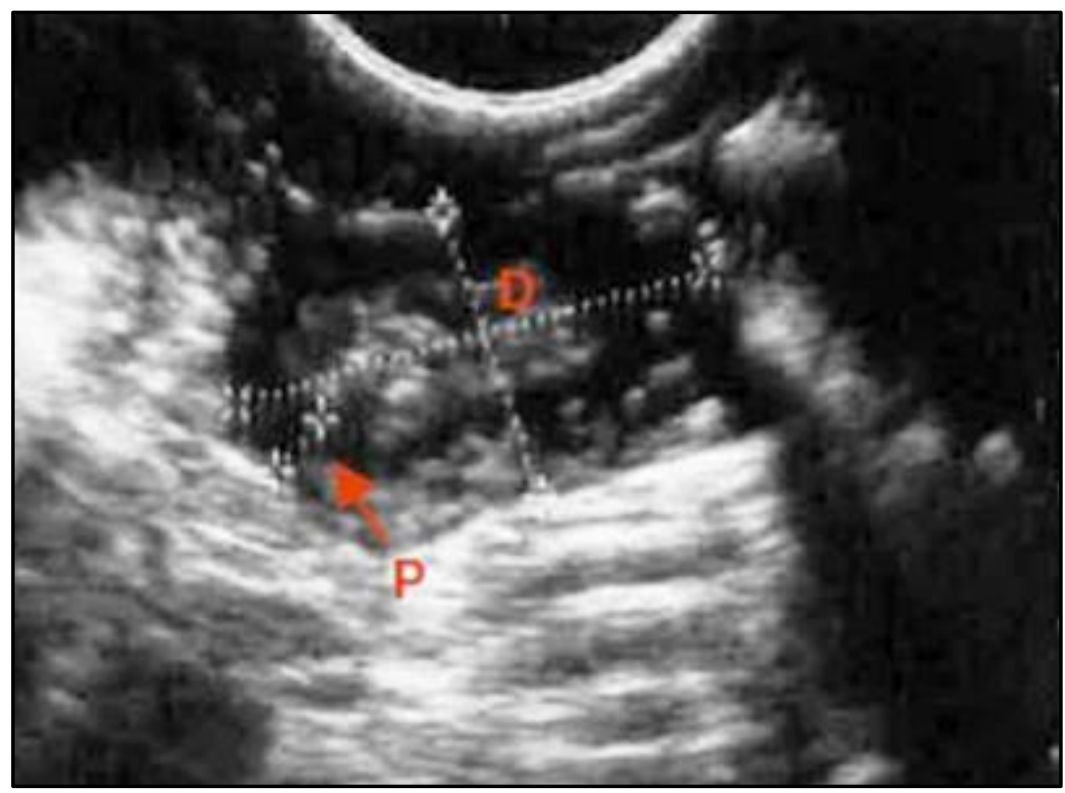

Figura 4: Imagem ultrassonográfica em corte transversal do corno uterino direito de cadelas no dia 0 (parto) (em corte transversal). O lume uterino apresenta grande quantidade de conteúdo líquido heterogêneo. $\mathrm{P}$ (parede uterina) $(0,52 \mathrm{~cm})$ DU (Diâmetro Uterino) $(3,72 \mathrm{~cm})$.

\section{RESULTADOS E DISCUSSÃO}

A maioria das cadelas do experimento apresentou-se receptiva ao tratamento, assim como aos exames ultrassonográficos, sendo que poucas fêmeas apresentaram ligeira resistência à manipulação (duas fêmeas). Ademais, salienta-se a facilidade para observação ultrassonográfica da silhueta uterina, corroborando com a descrição de Ferri \& Vicente (13), os quais consideram que, durante o puerpério, a observação do útero em cadelas é facilitada pelo aumento de volume e a boa diferenciação da estrutura em relação às adjacentes, propiciando avaliar sua arquitetura, ecogenicidade, características e diâmetro.

Os resultados dos achados ultrassonográficos de acordo com os grupos experimentais foram descritos na Tabela 1. Não foram verificadas diferenças significativas entre os resultados obtidos no experimento $(\mathrm{p}>0,05)$.

Tabela 1: Valores médios e de desvio padrão da espessura da parede uterina direita (PD) e esquerda (PE), lúmen uterino direito (LD) e esquerdo (LE), diâmetro uterino direito (DD) e esquerdo (DE) (cm) de cadelas em pós-parto eutócico no experimento, nos diferentes momentos do exame ultrassonográfico imediatamente após o parto - 0, e após $1,3,7$ e 14 dias

\begin{tabular}{clccccc}
\hline & \multirow{2}{*}{ Grupos } & \multicolumn{5}{c}{ Exames ultrassonográficos (dias) } \\
\cline { 3 - 6 } & & 0 & 1 & 3 & 7 & 14 \\
\hline \multirow{2}{*}{ PD } & 1 & $0,40 \pm 0,07$ & $0,36 \pm 0,08$ & $0,29 \pm 0,05$ & $0,25 \pm 0,07$ & $0,15 \pm 0,02$ \\
& 2 & $0,39 \pm 0,07$ & $0,35 \pm 0,05$ & $0,32 \pm 0,04$ & $0,27 \pm 0,05$ & $0,23 \pm 0,07$ \\
PE & 1 & $0,39 \pm 0,04$ & $0,37 \pm 0,06$ & $0,30 \pm 0,08$ & $0,26 \pm 0,07$ & $0,18 \pm 0,07$ \\
& 2 & $0,39 \pm 0,08$ & $0,35 \pm 0,06$ & $0,33 \pm 0,06$ & $0,27 \pm 0,06$ & $0,22 \pm 0,06$ \\
LD & 1 & $2,63 \pm 1,70$ & $2,52 \pm 1,87$ & $1,84 \pm 1,09$ & $1,72 \pm 0,91$ & $1,00 \pm 0,76$ \\
& 2 & $2,42 \pm 0,91$ & $2,08 \pm 0,92$ & $1,92 \pm 0,72$ & $1,57 \pm 1,18$ & $0,94 \pm 0,45$ \\
LE & 1 & $3,49 \pm 1,89$ & $2,99 \pm 1,63$ & $2,04 \pm 1,46$ & $1,71 \pm 0,96$ & $0,82 \pm 0,45$ \\
& 2 & $2,06 \pm 0,87$ & $2,41 \pm 1,16$ & $2,27 \pm 0,97$ & $1,43 \pm 0,79$ & $1,04 \pm 0,68$ \\
DD & 1 & $5,89 \pm 2,32$ & $5,84 \pm 3,10$ & $4,21 \pm 2,21$ & $3,27 \pm 1,26$ & $1,89 \pm 1,07$ \\
& 2 & $6,01 \pm 2,10$ & $5,26 \pm 2,05$ & $4,61 \pm 1,71$ & $3,22 \pm 1,81$ & $2,48 \pm 1,50$ \\
DE & 1 & $6,65 \pm 2,80$ & $6,50 \pm 2,96$ & $4,01 \pm 3,02$ & $3,04 \pm 1,63$ & $1,92 \pm 1,19$
\end{tabular}


2

$5,29 \pm 1,51$

$5,39 \pm 2,26$

$4,92 \pm 2,01$

$3,10 \pm 1,38$

$2,19 \pm 1,24$

Os resultados obtidos não diferiram significativamente pelo teste $\mathrm{F}$.

Observou-se que a espessura da parede uterina em ambos os cornos diminuiu gradativamente nos dois grupos experimentais até o $7^{\circ}$ dia de tratamento. Ao comparar os dois grupos, notou-se ao final dos tratamentos que G2 $(0,22 \pm 0,06 \mathrm{~cm})$ apresentava maior espessura de parede uterina que $\mathrm{G} 1(0,18 \pm 0,07 \mathrm{~cm})$. Esse fato reitera as afirmações de Ferri \& Vicente (13), que consideram a involução uterina um evento fisiológico que se completa em aproximadamente 12 semanas, embora o término dos eventos regenerativos teciduais seja às nove semanas, período em que as estruturas uterinas das cadelas estarão uniformemente hipoecoicos, tubulares e com diâmetro reduzido.

Neste estudo, as paredes uterinas direita e esquerda apresentaram diminuição da espessura de forma gradativa para os grupos G1 e G2, sendo qu entre os dias sete e 14 para $G$ a involução uterina mostrou-se acentuada, provavelmente devido ao tratamento utilizado. Esses resultados contrariam as citações de Ferri et al. (7) e Ferri \& Vicente (13), que afirmam que a musculatura uterina perde em poucos dias a capacidade de reagir a estímulos e de contrairse. Para tanto, cabe comentar que a eletroacupuntura gera estimulação elétrica nos tecidos tratados, produzindo efeitos cumulativos $(11,14,15)$.

Ao avaliar o lúmen uterino direito, pôde-se verificar, em ambos os grupos experimentais, uma diminuição gradativa até $\mathrm{o} 7^{\circ}$ dia (G1 - 1,72 $\pm 0,91 \mathrm{~cm}$ e G2 - $1,57 \pm 1,18 \mathrm{~cm}$ ), seguindo-se com a involução mais acentuada ao $14^{\circ}$ dia $(\mathrm{G} 1-1,00 \pm 0,76 \mathrm{~cm}$ e G2 $0,94 \pm 0,45 \mathrm{~cm})$. Para o lúmen uterino esquerdo, observou-se diminuição acentuada e uniforme para G1 e, em relação ao $\mathrm{G} 2$, houve uma tendência a tamanhos maiores (dias $1-2,41 \pm 1,16 \mathrm{~cm}$ e 3 $2,27 \pm 0,97 \mathrm{~cm})$ em comparação ao dia inicial $(2,06 \pm 0,87 \mathrm{~cm})$, involuindo nos dias $7(1,43 \pm 0,79 \mathrm{~cm})$ e $14(1,04 \pm 0,68 \mathrm{~cm})$.

A redução do diâmetro uterino direito mostrou-se uniforme para os dois grupos. Pôde-se verificar que, ao terceiro dia, o G2 apresentou taxa de involução menor $(4,61 \pm 1,71 \mathrm{~cm})$ que $\mathrm{G} 1$ $(4,21 \pm 2,21)$, tornando-se semelhantes ao $7^{\circ}$ dia (Tabela 1). Em relação ao diâmetro uterino esquerdo, os animais do grupo G1 apresentaram uma redução acentuada e gradativa entre os dias $1(6,50 \pm 2,96 \mathrm{~cm})$ e $3(4,01 \pm 3,02 \mathrm{~cm})$, seguindo-se involução uniforme nos dias subsequentes. O G2 apresentou ligeiro aumento do dia $0 \quad(5,29 \pm 1,51 \mathrm{~cm})$ comparando-se com o dia $1(5,39 \pm 2,26 \mathrm{~cm})$ pós-parto, reduzindo discretamente até o terceiro dia $(4,92 \pm 2,01 \mathrm{~cm})$.

Cabe comentar a importância da avaliação ultrassonográfica do diâmetro uterino em cadelas, no período de involução uterina. De acordo com Ferri \& Vicente (13), a determinação do diâmetro uterino é medida precisa para diagnosticar a involução uterina no puerpério fisiológico da cadela.

O grupo 1 apresentou involução uterina gradativa e uniforme entre os dias estudados, sendo verificada visualmente a descarga loquial constante para essas cadelas (sugerindo maior motilidade uterina). Esses resultados corroboram os de Dunn et al. (16), que avaliaram o efeito da estimulação elétrica transcutânea, em pontos de acupuntura, para incrementar as contrações uterinas em 20 mulheres com gestação a termo. Esses autores observaram aumento significativo na frequência e força das contrações uterinas, comparadas com as mulheres do grupo placebo. No presente trabalho, a involução uterina, de maneira geral, apresentou-se com maior uniformidade em G1 que G2, sugerindo que a utilização da acupuntura pode vir a auxiliar no controle de transtornos puerperais.

De acordo com Ferri et al. (7), ao início do período de pós-parto, a parede uterina apresenta-se espessada e irregular. Entre oito a 24 dias após o parto, a parede uterina apresenta-se mais fina e menos irregular, com conteúdo luminal, podendo se apresentar homogêneo, hipoecogênico ou mais ecogênico à medida que os líquidos são expelidos. Esses achados ultrassonográficos corroboram os resultados encontrados neste estudo ao avaliar as cadelas por meio da ultrassonografia.

Adicionalmente às alterações biométricas da parede e lúmen uterino das cadelas em fase pósparto, visibilizadas ao exame de ultrassom no presente estudo, são notórias as modificações do conteúdo uterino. Segundo Root \& Spaulding (17), entre um e quatro dias pós-parto, o conteúdo tem ecogenicidade mista (componentes ecogênicos como anecoicos), achados condizentes aos observados ao exames das cadelas estudadas.

\section{CONCLUSÕES}

Concluiu-se que a eletroacupuntura afeta positivamente o tônus uterino podendo corroborar para a prevenção de transtornos puerperais. Cabe lembrar que são poucas as informações literárias sobre o uso de medicinas complementares como a acupuntura e tratamento controle (método Sham), havendo a necessidade de maiores estudos.

\section{REFERÊNCIAS}


1. Scognamillo-Szabó MVR, Bechara GH. Acupuntura: histórico, bases teóricas e sua aplicação em Medicina Veterinária. Ciência Rural. 2010; 40: 491-500. Disponível em: http://dx.doi.org/10.1590/S0103-84782010005000004

2. Carneiro NM. Fundamentos da acupuntura médica. Florianópolis: Editora Sistema; 2001. p.709. Portuguese

3. Grunert E, Birgel EH. Obstetrícia veterinária. Porto Alegre: Sulina; 1989. p.129-135. Portuguese

4. Goericke-Pesch S, Schmidt B, Failing K, Wehrend A. Changes in the histomorphology of the canine cervix though the oestrus cycle. Theriogenology. 2010; 74:10751081. Disponível em: http://dx.doi.org/10.1016/j.theriogenology.2010.05.004.

5. Volpato R, Martins I, Ramos RS, Tsunemi MH, LauferAmorin R, Lopes MD. Imunoistoquímica de útero e cérvice de cadelas com diagnóstico de piometra. Arquivo Brasileiro de Medicina Veterinária e Zootecnia. 2012; 64: 1109-1117. Disponível http://dx.doi.org/10.1590/S0102-09352012000500004.

6. Pretzer SD. Clinical presentation of canine pyometra and mucometra: a review. Theriogenology. 2008; 70: 359363. Disponível em: http://dx.doi.org/10.1016/j.theriogenology.2008.04.028.

7. Ferri STS, Vicente WRR, Toniollo GH. Estudo da involução uterina por meio da ultra-sonografia (modo-B) em cadelas submetidas a cesariana. Arquivo Brasileiro de Medicina Veterinária e Zootecnia. 2003; 55. Disponível em: 09352003000200007.

8. Fayrer-Hosken RA, Mahaffey M, Miller-Liebl D, Candle AB. Early diagnosis of canine pyometra using ultrasonography. Veterinary Radiology \& Ultrasound. 1991; 32: 287-289. Disponível em: 10.1111/j.17408261.1991.tb00125.x.
9. Torro CA. Atlas prático de acupuntura. São Paulo: Varela; 1997. 185p. Portuguese.

10. Ding L. Acupuntura, teoria do meridiano e pontos de acupuntura. São Paulo: Editora Roca Ltda; 1996. 461p. Portuguese

11. Chan WW, Chen KY, Liu H, Wu LS, Lin JH. Acunputure for general veterinary practice. The Journal of Veterinary Medical Science. 2001; 63: 1057-1062. Disponível em: http://dx.doi.org/10.1292/jvms.63.1057.

12. Feliciano MAR, Muzzi LAL, Leite CAL, Junqueira MA. Ultra-sonografia bidimensional convencional, de alta resolução e tridimensional no acompanhamento da gestação em cadela. Arquivo Brasileiro de Medicina Veterinária e Zootecnia. 2007; 59: 1333-1337. Disponível em: 09352007000500037.

13. Ferri STS, Vicente WRR. Estudo ultra-sonográfico da involução uterina pós-parto em cadelas. Arquivo Brasileiro de Medicina Veterinária e Zootecnia. 2002; 54. Disponível em: http://dx.doi.org/10.1590/S010209352002000100003.

14. Stux G, Pomeranz B. Acupuncture textbook and atlas. Berlin: Springer Verlag; 1987. 105 p. English.

15. Altman S. Tecniques and instrumentation. In: Schoen AM. Veterinary acupunture. 2.ed. St Louis: Mosby; , 2001. p.102-108. English

16. Dunn PA, Rogers D, Halford K. Transcutaneous electrical nerve stimulation at acupuncturepoints in the induction of uterine contractions. Obstetrics \& Gynecology. 1989; 73: 286-290.

17. Root CR, Spaulding KA. Diagnostic imaging in companion animal theriogenology. Seminars in Veterinary Medicine and Surgery (Small Animal). 1994; 9: 7-27. 